\title{
Is there not a better way to prevent venousthromboembolism after total knee arthroplasty?
}

\author{
M. A. Snyder ${ }^{1}$, O. E. Vyrva ${ }^{2}$ \\ ${ }^{1}$ Trihealth Orthopedic \& Spine Institute, Cincinnati. USA \\ ${ }^{2}$ Sytenko Institute of Spine and Joint Pathology, Kharkiv. Ukraine
}

Background: aspirin at $325 \mathrm{mg}$ twice daily is now included as a nationally approved venous thromboembolism prophylaxis protocol for low risk total knee arthroplasty patients. The purpose of this study is to examine if there is a difference in deep vein thrombosis occurrence after a limited tourniquet total knee arthroplasty using aspirin-based prophylaxis with or without extended use of mechanical compression device therapy. Methods: one hundred limited tourniquet total knee arthroplasty patients, whose deep vein thrombosis risk was managed with aspirin $325 \mathrm{mg}$ twice daily for 3 weeks, were randomized to either utilizing an mechanical compression device during hospitalization only or extended use at home up to six weeks post-op. Lower extremity Duplex venous ultrasonography was completed on the second post-op day, 14 days postop, and at 3 months post-op to confirm absence of deep vein thrombosis after treatment. Results: the deep vein thrombosis rate for the post-discharge mechanical compression device therapy group was $0 \%$ and $23.1 \%$ for the inpatient mechanical compression device group $(p<0.001)$. All deep vein thrombosis resolved by 3 months postop. Patient satisfaction was $9.56 \pm 0.82$ for post-discharge mechanical compression device patients versus $8.50 \pm 1.46$ for in patient mechanical compression device patients $(p<0.001)$. Conclusion: limited tourniquet total knee arthroplasty patients that were mobilized early, managed with Aspirin for 3 weeks post-op, and mechanical compression device therapy for up to 6 weeks post-op experienced superior deep vein thrombosis prophylaxis than patients receiving mechanical compression device therapy only as an inpatient ( $p<0.05)$. The $0 \%$ incidence of non-symptomatic deep vein thrombosis prevented by Aspirin and extended use mechanical compression device further validates this type of prophylaxis in low deep vein thrombosis risk total knee arthroplasty patients. Key words: total knee arthroplasty, aspirin, mechanical compression mobilization.
В настоящее время аспирин в дозе 325 мг два раза в день включен в национальный протокол США как профилактика венозной тромбоэмболии у пациентов с низким риском развития ее после тотального эндопротезирования коленного сустава (ТЭКС). Цель: изучить различия развития тромбоза глубоких вен (ТГВ) у больных после ТЭКС, получавших аспирин в качестве профилактики, при ограниченном по времени использовании турникета с длительным применением или без устройства для механической компрессионной терапии (УМКТ). Методь:: в исследование включено 100 пациентов, принимавщих профилактически аспирин 325 мг дважды в день в течение 3 недель. Больных рандомизировали на 2 группы в зависимости от длительности применения УМКТ: I - только в течение стационарного лечения, II-c продолжением в домашних условиях до 6 недель после операции. Дуплексное ультразвуковое исследование вен нижних конечностей всем пациентам проводили на 2 и 14-й дни, через 3 мес. после операции для оценки ТГВ. Результаты: частота ТГВ в группе II составила 0\%, а в I- 23,1\% ( $<<0,001)$. Все пачиенты анкетированы и обследованы через 3 мес. после операции. Степень удовлетворенности больных группы II составила 9,56 \pm 0,82, а группы I (периодом с использованием УМКТ только в стационаре) $-8,56 \pm 1,46(p<0,001)$. Выводы: пацииенты с лимитированным во времени использованием турникета при ТЭКС, ранней послеоперационной мобилизацией, профилактическим применением аспирина 325 мг дважды в день в течение 3 недель и использованием УМКТ до 6 недель после операчии имели значительно более низкий уровень развития ТГВ, чем те, которым применялось УМКТ только в стационаре $(p<0,05)$. Кроме того, $0 \%$ случаев бессимптомных ТГВ, предотвращенных аспирином и длительным использованием УМКТ, также подтверждает высокую эффективность этого вида профилактики венозной тромбоэмболии у больных с низким риском ее развития после ТЭКС. Ключевые слова: тотальное эндопротезирование коленного сустава, аспирин, механическая компрессионная мобилизачия.

Key words: total knee arthroplasty, aspirin, mechanical compression mobilization 


\section{Introduction}

Among total joint surgery complications, the risk of venous thromboembolism (VTE) is of the utmost concern warranting VTE prophylaxis in even low risk patients. Patients undergoing total knee arthroplasty (TKA) are particularly at risk for VTE with an incidence rate of $17-53 \%$ depending on the method of prevention [1] therein warranting routine prevention in even low-risk patients [2-7]. Standard of care guidelines implemented by the American Academy of Orthopedic Surgeons (AAOS) has mandated a regimen of thromboprophylaxis for all total hip or knee replacement patients, and has outlined those regimens with updated research evidence, taking into account the patient's history and risk of thrombosis [2]. The 2014 guidelines on VTE management are the result of the Surgical Care Improvement Project (SCIP), a national partnership of organizations including the Joint Commission and the Centers for Medicare and Medicaid Services, with the goal of reducing surgical complications and publishing a uniform set of national hospital quality measures. This goal is currently limited by the lack of conclusive evidence that would allow the endorsement of one regimen over another, and so the prevention method of choice remains controversial [2]. The conflicting evidence is well documented in the AAOS Guidelines citing such reasons as lack of standardized drug doses, unstandardized routes of administration, un-standardized durations of therapy, a dearth of placebo controlled studies, as well as nonrepresentative research populations, or underpowered studies [2].

The AAOS guidelines have been revised to include aspirin in the list of acceptable thromboprophylactic regimens [2]. In a large level II evidence registry study, 3060 total joint replacement patients receiving at least 10 days of $\mathrm{MCD}$ with or without aspirin were found to have similar incidence of VTE to patients receiving standard chemoprophylactics [3]. The AAOS recognizes that a surgeon may prefer to administer the safe and convenient aspirin over other common chemoprophylactic agents [7].
When using aspirin, the AAOS advises the addition of a mechanical compression device (MCD) to increase the efficacy of aspirin in VTE prevention [2].

MCDs have an assortment of modalities (figure) such as pneumatic compression or sequential compression; and while there is inconclusive evidence as to effectiveness between processes, MCDs are thought to influence Virchow's triad by reducing venous stasis, thereby reducing the incidence of DVT or PE $[7,8]$. When used in combination with the AAOS indicated antiplatelet agent, aspirin, adequate thromboprophylaxis may be obtained reducing the risk of debilitating side effects or severe bleeding associated with warfarin and other chemoprophylactics.

Differences among MCD products, or what modality of compression they employ, do not appear to have an impact on efficacy of thromboprophylaxis. The most marked indication of thromboprophylaxis is patient compliance, and the location of the compression [8]. Thus, ease of operation, reliability, and the ability to gauge compliance may be the most relevant features of an effective MCD.

The Cothera VPULSE ${ }^{\mathrm{TM}}$ Compression and Cold Therapy System (Plano, TX; United States: Cothera, LLC) was FDA approved in 2013, and is designed for home use allowing extended post-operative therapy. The VPULSE ${ }^{\mathrm{TM}}$ device has the ability to provide intermittent sequential pneumatic compression for the prevention of venous thrombosis related to hospitalization [9], therein fulfilling the AAOS requirements following TKA [2]. The device is designed to be user-friendly, and records usage data which will be instrumental for monitoring patient compliance for the purposes of this study [9].

As a multifactorial disease, VTE onset may occur during the knee surgery through periods of high flexion and tourniquet use, thus VTE prophylaxis safety and efficacy may be maximized by the implementation of a multimodal thromboprophylactic regimen $[7,10,11]$. Multimodal enhancements strengthen protocols for venous thromboembolism prevention in TKA patients.
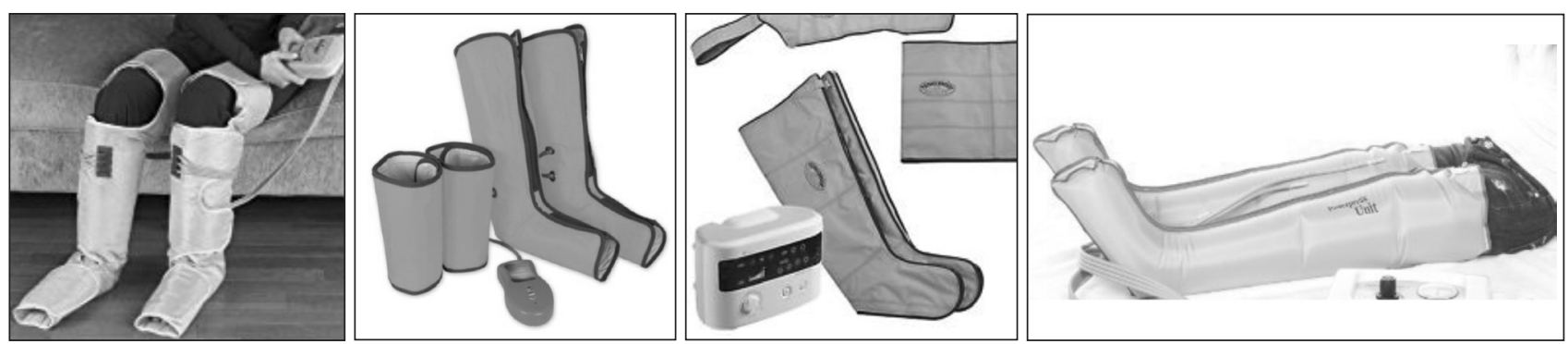

Figure. Different types of mechanical comperession devices 
The use of a tourniquet during the TKA procedure results in venous stasis, trauma to the endothelium of deep veins in the leg, hypoxia of the leg, and increased clotting factors upon release of the tourniquet $[7,11]$. When compared with standard tourniquet utilization during TKA, a minimized tourniquet technique is associated with a lower rate of VTE [12]. Recent evidence suggests that postoperative recovery and early range of motion (ROM) of the knee may be superior in a minimized tourniquet procedure [13]. Lack of mobilization may facilitate venous stasis, and as such is a contributing factor for DVT, which makes early mobilization of the patient crucial in a multimodal regimen [14]. This study attempts to provide a randomized control trial providing clarification on a multi-modal VTE approach that includes rapid postoperative mobilization of the patient, limiting the use of a tourniquet to no more than five minutes during cementation, prophylactic aspirin, and MCD therapy.

\section{Materials and methods}

This study was an IRB approved, prospective, randomized, control trial conducted in Cincinnati Ohio. Primary total knee arthroplasty patients, 18 years or older were included if they were determined to be at low risk for VTE. Patients were excluded if they had a high risk BMI of greater than $40 \mathrm{~kg} / \mathrm{m}$, an American Society of Anesthesiologist (ASA) score greater than III, suffer from NSAID intolerance, or any orthopaedic or medical comorbidity that would prevent postoperative rapid mobilization and compliance with MCD use. All subjects consented into the study were randomized by the research coordinator in a 1:1 ratio to either Group A or Group B by a permuted mixed block size randomization table. Group A served as the control group, only receiving MCD therapy while an inpatient following total knee arthroplasty. Group B, the experimental group, continued the MCD therapy for up to 6 weeks following discharge from the hospital. All patients underwent a multimodal VTE prophylactic regimen consisting of administration of 1 gram pre-operative Tranexamic Acid, and limiting tourniquet application to a maximum of five minutes only during exsanguination to improve cementation and to minimize blood loss. Early rapid mobilization was facilitated, and all subjects received prophylactic aspirin at $325 \mathrm{mg}$ twice daily for 3 weeks immediately postoperatively. To standardize the therapy among groups, the MCD utilized was the Cothera VPULSE ${ }^{\mathrm{TM}}$ for all study patients. Use of the MCD was initiated immediately post-operatively, and continued for at least 3 weeks, and up to 6 weeks depending on when the patients had their second post-operative visit scheduled.

Post-operatively, bilateral venous duplex ultrasounds (LEDVU) were conducted on all patients two days post-operatively and at 2 weeks (14-19 days) post-operatively to detect the incidence of DVT. Patients were seen $(10 \pm 7)$ days post-operatively in the surgeon's office for routine follow up knee exam. Patients were also seen at 3-6 weeks postoperatively for routine follow up and to complete an overall satisfaction assessment. The VPULSE ${ }^{\mathrm{TM}}$ data chip was collected for recording the total number hours of MCD usage. Length of hospital stay (LOS), 30-day adverse events were recorded, and overall patient satisfaction was evaluated with a 10 point Likert scale [15].

The 10 point Likert scale was determined to be the best way to keep track of patient satisfaction because of its consistency and reliability in conveying patient responses. Ten points were chosen to try and maximize reliability, validity and discriminating power without comprising consistency or test-retest reliability [16]. On the last study visit of each patient, the study coordinator asked each participant, «Overall on a scale of 1 to 10 , where 1 is very dissatisfied, 5 is neutral, and 10 is very satisfied, where would you rate your satisfaction with the total knee arthroplasty you have received from the start of the study to today?». Although the study coordinator was not able to be blinded to the randomization groups, the question was asked in exactly the same way with each patient. There was no leading of the patient, and no further inquiry into the patient's satisfaction to avoid leading the study subject into a higher satisfaction score. These patient reported satisfaction scores were collected to ascertain whether the patient associated the MCD usage as a burden for his or her overall patient experience.

The sample size was determined to be 100 patients based on a meta-analysis comparing DVT incidence among four commonly ascribed treatment regimens [1], understanding that no prior studies had combined the same regimen of $325 \mathrm{mg}$ aspirin twice daily for 3 weeks post-operatively in conjunction with MCD therapy. The assumption was made that this sample size would suffice for a medium effect size at $80 \%$ power and alpha $<0.05$. DVT incidence rate was determined to be the primary outcome, as analyzed by a univariate chi-square analysis.

All statistical analyses were performed using IBM SPSS Statistics for Windows version 21 (Armonk, NY: IBM Corp). Demographic variables included patient age, gender, race, anesthesia type, ASA score, 
BMI, surgery side, and associated comorbidities (table 1). Secondary clinical outcome variables included length of stay (LOS), tourniquet time, estimated blood loss (EBL), hemoglobin, hematocrit, days per week of physical therapy, hours of continuous passive motion (CPM), MCD usage type and hours, adverse events and overall satisfaction (table 2). A univariate chi-square analysis or Fisher's exact test was employed to compare the Post-discharge VPULSE group with the Inpatient VPULSE group on DVT occurrence at post-operative day two and at two weeks postoperatively. An Independent samples/test was performed on all normally distributed data and a non-parametric Mann-Whitney U test was utilized for non-normally distributed data. All p-values were one-tailed and $\mathrm{p}$-value $<0.05$ was considered statistically significant.

\section{Results}

A total of 163 patients were screened and after meeting the study eligibility, 100 patients enrolled in the study and were randomized either to the inpatient VPULSE group $(n=52)$ or to the post-discharge VPULSE group $(n=48)$. Recruitment and follow up started in April 2014 until January 2015, and follow-up continued until all patients had completed the study in February 2015. All patients mean age was $(62.76 \pm 9.24)$ with the post-discharge VPULSE group mean age as $(59.85 \pm 8.20)$, and the inpatient VPULSE group mean age as $(65.44 \pm 9.40)$ ( $\mathrm{p}=0.002$, statistically significant). The study population was $40 \%$ male, and $60 \%$ female with $35.4 \%$ $(\mathrm{n}=17)$ male in the post-discharge VPULSE group, and $44.2 \%(n=23)$ male in the inpatient VPULSE group, and $64.6 \%(\mathrm{n}=31)$ female in the post-discharge VPULSE group, and $55.8 \%(n=29)$ female in the inpatient VPULSE group. A significantly decreased risk of DVT was observed at both postoperative day 2 and week 2 in the post-discharge VPULSE group compared to the inpatient VPULSE group (0\% vs $11.5 \%, n=6, p=0.017$ ).

Subject demographics

\begin{tabular}{|c|c|c|c|c|}
\hline Index & Total ( $\mathrm{N}=100)$ Mean (SD); n (\%) & $\begin{array}{l}\text { Post-discharge VPULSE } \\
(\mathrm{n}=48) \text { Mean (SD); n (\%) }\end{array}$ & $\begin{array}{c}\text { Inpatient VPULSE } \\
(\mathrm{n}=52) \text { Mean (SD); } \mathrm{n}(\%)\end{array}$ & P-value \\
\hline AGE (years) & $62.76(9.24)$ & $59.85(8.20)$ & $65.44(9.40)$ & 0.002 \\
\hline \multicolumn{5}{|l|}{ GENDER } \\
\hline Male & $40(40.0)$ & $17(35.4)$ & $23(44.2)$ & \multirow{2}{*}{0.369} \\
\hline Female & $60(60.0)$ & $31(64.6)$ & $29(55.8)$ & \\
\hline RACE & & $51(100)$ & $48(100)$ & \multirow{4}{*}{0.367} \\
\hline Caucasian & $98(98.0)$ & 47 (97.9) & $51(98.1)$ & \\
\hline African American & $1(1.0)$ & $1(2.1)$ & $0(0.00)$ & \\
\hline Other & $1(1.0)$ & $0(0.00)$ & $1(1.9)$ & \\
\hline BMI & $30.09(4.22)$ & $30.85(4.17)$ & $29.38(4.18)$ & 0.081 \\
\hline \multicolumn{5}{|l|}{ COMORBIDITY } \\
\hline Hypertension (yes) & $39(39.0)$ & $17(35.4)$ & $22(42.3)$ & 0.480 \\
\hline CAD (yes) & $4(4.0)$ & $3(6.3)$ & $1(1.9)$ & 0.279 \\
\hline Diabetes (yes) & $8(8.0)$ & $2(4.2)$ & $6(11.5)$ & 0.162 \\
\hline \multicolumn{5}{|c|}{ ANESTHESIA TYPE } \\
\hline General & $11(11.0)$ & $6(12.5)$ & $5(9.6)$ & \multirow{3}{*}{0.645} \\
\hline Spinal & $89(89.0)$ & $42(87.5)$ & $47(90.4)$ & \\
\hline Other & $1(1.0)$ & $0(0.00)$ & $1(1.9)$ & \\
\hline \multicolumn{5}{|l|}{ ASA SCORE } \\
\hline 1 & $7(7.0)$ & $4(8.3)$ & $3(5.8)$ & \multirow{4}{*}{0.711} \\
\hline 2 & $62(62.0)$ & $29(60.4)$ & $33(63.5)$ & \\
\hline 3 & $30(30.0)$ & $14(29.2)$ & $16(30.8)$ & \\
\hline 4 & $1(1.0)$ & $1(2.1)$ & $0(0.00)$ & \\
\hline \multicolumn{5}{|l|}{ SURGERY } \\
\hline Left TKA & $56(56.0)$ & $27(56.3)$ & $29(55.8)$ & \multirow{2}{*}{0.961} \\
\hline Right TKA & $44(44.0)$ & $21(43.8)$ & $23(44.2)$ & \\
\hline
\end{tabular}


Patient Outcomes and Clinical Data

\begin{tabular}{|l|c|c|c|c|}
\hline \multicolumn{1}{|c|}{ Index } & Total (N=100); $(\%)$ & $\begin{array}{c}\text { Post-discharge VPULSE } \\
(\mathrm{n}=48) ; \mathrm{n}(\%)\end{array}$ & $\begin{array}{c}\text { Inpatient VPULSE } \\
(\mathrm{n}=52) ; \mathrm{n}(\%)\end{array}$ & P-value \\
\hline Total DVTs ¥ & $12(12.0)$ & $0(0.0)$ & $12(23.1)$ & $<0.001$ \\
\hline DVT Postop Day 2¥ & $6(6.0)$ & $0(0.0)$ & $6(11.5)$ & 0.017 \\
\hline DVT Post op Day 14 $¥$ & $6(6.0)$ & $0(0.0)$ & $6(11.5)$ & 0.017 \\
\hline Length of Stay (days) & $2.12(0.33)$ & $2.06(0.25)$ & $2.17(0.38)$ & 0.086 \\
\hline Tourniquet Time (min) & $5.18(0.95)$ & $5.27(0.95)$ & $5.08(0.94)$ & 0.329 \\
\hline Estimated Blood Loss (mL) & $89.00(27.60)$ & $89.06(32.58)$ & $88.94(22.37)$ & 0.684 \\
\hline Hemoglobin (g/dL) & $11.33(1.35)$ & $11.25(1.05)$ & $11.41(1.58)$ & 0.543 \\
\hline Hematocrit (\%) & $32.76(3.22)$ & $32.85(3.07)$ & $32.67(3.38)$ & 0.78 \\
\hline Physical Therapy (Days per Week) & $2.62(0.49)$ & $2.63(0.49)$ & $2.62(0.49)$ & 0.922 \\
\hline CPM* (Hours per day) & $5.07(0.99)$ & $5.19(1.09)$ & $4.96(0.90)$ & 0.269 \\
\hline MCD usage & & & & \\
\hline VPulse SC (hours)* & $91.40(72.58)$ & $147.71(68.87)$ & $39.43(10.77)$ & $<0.001$ \\
\hline VPulse Cooling (hours)* & $91.90(74.69)$ & $149.79(71.16)$ & $38.47(9.85)$ & $<0.001$ \\
\hline VPulse DC (hours)* & $76.90(63.57)$ & $120.32(67.87)$ & $36.04(8.89)$ & $<0.001$ \\
\hline Adverse Events** & $2(2.0)$ & $1(1)$ & $1(1.0)$ & 0.536 \\
\hline Overall Patient Satisfaction *** (NRS) ${ }_{\mathrm{w}}^{*}$ & $9.01(1.31)$ & $9.56(0.82)$ & $8.50(1.46)$ & $<0.001$ \\
\hline
\end{tabular}

$¥$ Chi-square analysis.

* Mann-Whitney U-test.

** DVTs were not included as adverse events since the incidence of DVT was analyzed separately.

*** Independent Samples t-test.

The total incidence of DVT was $23.1 \%$ in the inpatient VPULSE group $(n=12)$, and $0 \%$ in the postdischarge VPULSE group $(\mathrm{p}<0.001)$. VPULSE ${ }^{\mathrm{TM}}$ usage time was tested using non-parametric analysis, specifically the independent-samples Mann-Whitney $\mathrm{U}$ test and a statistically significant difference was found in the post-discharge VPULSE group versus the inpatient VPULSE group, that is, patients used the machine significantly longer in the postdischarge VPULSE group. Finally, an independentsamples t-test demonstrated a significant increase in patient satisfaction in the post-discharge VPULSE group $(9.56 \pm 0.82)$ versus the inpatient VPULSE group $(8.50 \pm 1.46)$. This difference was significant at the $\mathrm{p}<0.001$ level (Table 2).

The absolute risk reduction (ARR), also termed the risk difference, was calculated by subtracting the proportion of patients with DVT in the post-discharge VPULSE group from that in the inpatient VPULSE group. Since $11.5 \%(n=6)$ of the patients developed DVT in the inpatient VPULSE group, with no patients developing DVT in the post-discharge VPULSE group after day 2 and week 2 of observation, the absolute risk reduction was $11.5 \%$ at each time point. Additionally, the relative risk reduction (RRR), calculated as the proportion of the ARR and the event rate in the control group, was $100 \%$, with the overall ARR at $23.1 \%$.

\section{Discussion}

The results of this study demonstrate the utility of implementing an extended use MCD in the prevention of DVT incidence, and provide further evidence that a prophylactic regimen of aspirin and MCD therapy might be at least as effective as other comparable methods of thromboprophylaxis. The multimodal approach of limiting the use of a tourniquet to no more than five minutes, rapid post-operative patient mobilization and $325 \mathrm{mg}$ aspirin twice daily for 3 weeks postoperatively in combination with MCD therapy demonstrated a low non-symptomatic incidence rate (12\%), and an even lower symptomatic DVT incidence rate $(1 \%)$, while minimizing bleeding complications $(1 \%)$. Aspirin is a generally safe, inexpensive and readily available thrombolytic agent, and when used in combination with at least 3 weeks of MCD therapy demonstrates superiority to the standard treatment of MCD therapy only during the inpatient stay following TKA (0 \% DVT incidence compared to $12.0 \%$ DVT incidence). Low-risk VTE patients may benefit from the findings in this study, as the side effects of concurrent aspirin and extended MCD use 
minimized bleeding complications $(0 \%$ in the postdischarge VPULSE patients) This awareness of a safe alternative to disproportionate use of high risk chemoprophylactics contributes to the future development of effective VTE prevention in total joint arthroplasty guidelines.

Reitman et al. examined the effects of a multimodal approach utilizing intraoperative heparin prior to tourniquet inflation, hypotensive epidural anesthesia, inpatient use of pneumatic compression boots, and 6 weeks of aspirin (325 mg twice daily), and reported an overall DVT rate of $4.0 \%(n=954$ TKA patients). However, ultrasonography was only performed in this study population at the time of discharge, and they reported a longer average length of stay (4.47 days versus $(2.12 \pm 0.33)$ ), which makes the DVT incidence rate difficult to compare. It also suggests evidence that the longer hospital stay, and consequently the longer use of the MCD therapy might have decreased the DVT incidence rate in this study [7]. Further, only performing ultrasonography at the time of discharge would have missed the $6 \%$ incidence of DVT in the inpatient VPULSE patients that developed DVT 2 weeks post-operatively, thus making the discordant DVT incidence rates less significant (4\% versus $6 \%$ inpatient VPULSE group, and $0 \%$ postdischarge VPULSE group).

Although pre-operative baseline scans were not performed, the post-operative day 2 scan served as a baseline, since all patients were receiving equivalent treatments until this point. Following discharge, the post-discharge VPULSE group experienced no DVTs $(n=0)$, while the inpatient VPULSE group experienced the $6 \%$ incidence of DVT $(n=6)$. That DVT occurred prior to the experimental treatment in half of the patients found to develop a DVT, may be attributed to an inherent increased risk for developing

DVT in these patients. Although every effort was made to include only patients at low risk of developing DVT by identifying contributing factors preoperatively, these patients may have had an unknown slightly increased risk of DVT. Patients may not have known or reported certain risk factors such as an unknown family history of DVT, or an unknown vein condition.

Overall, only 1 patient $(1 \%)$ was symptomatic for DVT developing mild pain in the calf of the operative leg at his post-operative day 2 scan, and this DVT persisted to his 2 week post-operative LEDVU scan (inpatient VPULSE ${ }^{\mathrm{TM}}$ group). The efficacy of this multimodal thromboprophylactic regimen becomes evident when compared to a $0.92 \%$ incidence rate of 3060 TKA and THA patients symptomatic for VTE (and confirmed by imaging) [3], as well as the superiority of the post-discharge VPULSE ${ }^{\mathrm{TM}}$ group's outcome. The low overall incidence of VTE (23.1\% in the inpatient VPULSE group; and $0 \%$ in the post-discharge VPULSE group) in this study is significantly lower than the estimated non-symptomatic DVT incidence rate of $17-53 \%$ in TKA patients without optimal prophylaxis [1].

The mean hospital length of stay was 2 days, and the complication rate was $2 \%(n=2)$. One such complication was a gastrointestinal (GI) bleed occurring after the patient had been placed on enoxaparin to alleviate a DVT found two weeks postoperatively (inpatient VPULSE group), and the other adverse event was an ER visit due to an acute case of gastritis (postdischarge VPULSE group), which was not definitively related to the procedure or aspirin administration, since all patients were treated with $10 \mathrm{mg}$ famotidine for up to 6 weeks.

The laterality of the DVTs that were found were primarily located in the operative limb, although 2 patients had DVTs in the non-operative limb, and not in the operative limb. One patient had a right knee TKA, and developed a right soleal vein DVT, while the other patient had a right knee TKA, and was found to have a left soleal vein DVT. These two patients were in the inpatient VPULSE group and the DVTs were only identified during the 2 week postoperative LED scan. Of those DVTs that were located in the operative limb, 3 were found in the popliteal vein, 7 - in the soleal vein, 2 - in an unspecified location of the lower extremity, and 1 patient had a total occluding saphenous vein DVT. All DVTs, except for those found in 1 patient, found at the post-op day 2 scan persisted to the post-op week 2 scan. Once identified, all DVTs were treated with either warfarin or rivaroxaban until resolved. No transfusions were performed, no patients were readmitted, and all DVTs were resolved by the 3 month follow-up knee exam, as verified by LEDVU.

The multimodal regimen utilized in this study was chosen for its low risk and seeming high efficacy, however Parvizi et al. presented the efficacy of a similar study utilizing only $81 \mathrm{mg}$ of aspirin twice daily concurrently with inpatient MCD therapy.

This prospective study found no difference in VTE incidence between the high dose and low dose aspirin group ( $0 \%$ versus $0.2 \%)$, and a decrease in GI bleeding among the low-dose aspirin patients [17]. Since low-dose aspirin is found to have sufficient antiplatelet properties, this would encourage a follow-up study with low-dose aspirin, MCD use, and LEDVU 
imaging to establish an equivalent or superior nonsymptomatic DVT rate.

Overall satisfaction was statistically higher $(\mathrm{p}<0.001)$ among the post-discharge VPULSE group $(9.56 \pm 0.82)$ than the inpatient VPULSE group $(8.50 \pm 1.46)$. While patient satisfaction was hypothesized to be statistically insignificant between the groups, the higher satisfaction may be an indication of improved satisfaction due to patient activation. Evidence suggests higher satisfaction scores when patients are involved in their own health improvement [18], such as compliant MCD usage. If the study were able to be blinded, then this hypothesis may be able to be supported or refuted, which cannot be done at this time. This study was only able to provide evidence that overall satisfaction scores with the patient experience was not compromised by instructing the patient to use the MCD consistently and reliably throughout the 3 week post-operative period.

The younger patient population in the post-discharge VPULSE group may also be attributed, at least in part, to the superior overall satisfaction in this group, however, this finding has not been supported by the literature. Although not well understood, younger patients are considered a high risk patient population for reporting lower satisfaction scores following total knee arthroplasty $[19,20]$, which was not reflected in this study. The mechanism behind the improved patient satisfaction requires further investigation, perhaps through the use of a blinded post-operative assessor rather than the study coordinator to prevent bias, or even through a more comprehensive satisfaction questionnaire.

Some weaknesses of this study include the data usage chip of the MCD. The post-discharge VPULSE group did use the sequential compression (SC) setting of the MCD more hours total than the inpatient VPULSE group ((147.71 \pm 68.87$)$ versus $(91.40 \pm 72.58)$, $\mathrm{p}<0.001$ ), however the data chip of the MCD did not allow the researchers to examine how this usage was spread over the 3 weeks. This means that patient compliance in the post-discharge VPULSE group may have dropped off significantly following the first several days post-discharge.

Although this study could be improved by further describing patient compliance, this observation may be offset by the role of rapid mobilization that all patients underwent. In this way the MCD therapy may act as a bridge between the inpatient hospital stay, and the length of time before resumption of a patient's activities of daily living (ADLs) where venous stasis is mitigated, and the elements of Virchow's triad are interrupted [21]. The multimodal approach utilized in this study complemented the design of the clinical trial to minimize any conflicting factors, such as the varying hours of MCD therapy completed each day.

While the implementation of post-discharge VPULSE $^{\mathrm{TM}}$ MCD therapy was able to entirely reduce the risk of DVT for the patients in the post-discharge VPULSE group (Relative risk reduction - $100 \%$ ) eliminating the incidence in DVT among the 48 patients randomized to extended use of the device, this study did not establish the best VTE prevention protocol. However, additional examinations of the use of aspirin in conjunction with MCD therapy may reinforce the findings of this study and lead to the creation and subsequent implementation of optimized regimens that offer low incidence of VTE, and fewer bleeding and surgical wound complications in postoperative TKA patients.

Conflict of interest. The authors declare the absence of conflict of interest.

\section{References}

1. Meta-analysis of thromboembolic prophylaxis after total knee arthroplasty / G. H. Westrich, S. B. Haas, P. Mosca, M. Peterson // J. Bone Joint Surg. Br. — 2000. — Vol. 82 (6). - P. 795-800.

2. Preventing venous thromboembolic disease in patients undergoing elective hip and knee arthroplasty. Evidence-based guideline and evidence report [online]. $-2^{\text {nd }}$ ed. - American Academy of Orthopaedic Surgeons, 2011. — Available from : http://www. aaos.org/research/guidelines/VTE/VTE_full_guideline.pdf.

3. A mobile compression device for thrombosis prevention in hip and knee arthroplasty / C. W. Colwell Jr., M. I. Froimson, S. D. Anseth [et al.] // J. Bone Joint Surg. Am. — 2014. Vol. 96 (3). — P. 177-183. — DOI: 10.2106/JBJS.L.01031.

4. Aspirin and warfarin for thromboembolic disease after total joint arthroplasty / P. A. Lotke, H. Palevsky, A. M. Keenan [et al.] // Clin. Orthop. Relat. Res. — 1996. — № 324. P. 251-258.

5. A systematic review on the effect of aspirin in the prevention of post-operative arterial thrombosis in patients undergoing total hip and total knee arthroplasty / F. Lussana, A. Squizzato, E. T. Permunian, M. Cattaneo // Thromb. Res. - 2014. Vol. 134 (3). — P. 599-603. — DOI: 10.1016/j.thromres.2014.06.027.

6. Venous thromboembolic disease after total hip and knee arthroplasty: current perspectives in a regulated environment / V. D. Pellegrini Jr., N. E. Sharrock, G. D. Paiement [et al.] // Instr. Course Lect. - 2008. - Vol. 57. - P. 637-661.

7. A multimodality regimen for deep venous thrombosis prophylaxis in total knee arthroplasty / R. D. Reitman, R. H. Emerson, L. L. Higgins, T. R. Tarbox // J. Arthroplasty. — 2003. Vol. 18 (2). - P. 161-168. — DOI: 10.1054/arth.2003.50026.

8. Morris R. J. Evidence-based compression: prevention of stasis and deep vein thrombosis / R. J. Morris, J. P. Woodcock // Ann. Surg. — 2004. — Vol. 239 (2). — P. 162-171. DOI: 10.1097/01.sla.0000109149.77194.6c.

9. Review: Cothera VPULSE compression and cold therapy system for prevention of deep vein thrombosis and pulmonary embolisms [online]. - Medgadget, LLC. 2013. - Available from: http://www.medgadget.com/2013/12/review-cotheravpusle-compression-and-cold-therapy-system-for-preventionof-deep-vein-thrombosis-and-pulmonary- embolisms.html.

10. Lachiewicz P. F. Multimodal prophylaxis for THA with mechanical compression / P. F. Lachiewicz, E. S. Soileau // Clin. 
Orthop. Rela.t Res. - 2006. - Vol. 453. - P. 225-230. DOI: 10.1097/01.blo.0000238861.84733.9d.

11. Lu N. Multimodal prophylaxis for venous thromboembolic disease after total hip and knee arthroplasty: current perspectives / N. Lu, E. A. Salvati // Chin. J. Traumatol. — 2010. Vol. 13 (6). - P. 362-369.

12. A comparison of outcomes with and without a tourniquet in total knee arthroplasty: a systematic review and meta-analysis of randomized controlled trials / I. Alcelik, R. D. Pollock, M. Sukeik [et al.] // J. Arthroplasty. — 2012. — Vol. 27 (3). P. 331-340. — DOI: 10.1016/j.arth.2011.04.046.

13. Faster recovery without the use of a tourniquet in total knee arthroplasty / A. Ejaz, A. C. Laursen, A. Kappel [et al.] // Acta Orthop. - 2014. - Vol. 85 (4). - P. 422-426. DOI: $10.3109 / 17453674.2014 .931197$.

14. Safety and efficacy of multimodal thromboprophylaxis following total knee arthroplasty: a comparative study of preferential aspirin vs. routine coumadin chemoprophylaxis / M. W. Gesell, A. Gonzalez Della Valle, S. Bartolome Garcia [et al.] // J. Arthroplasty. — 2013. — Vol. 28 (4). — P. 575-579. DOI: $10.1016 /$ j.arth.2012.08.004.

15. Likert R. A technique for the measurement of attitudes / R. Likert. — New York : The Science Press, 1932. - 55 p.

16. Preston C. C. Optimal number of response categories in rating scales: reliability, validity, discriminating power, and respondent preferences / C. C. Preston, A. M. Colman // Acta Psychol. (Amst.). — 2000. — Vol. 104 (1). — P. 1-15.

17. Low dose aspirin: an effective chemoprophylaxis for preventing venous thromboembolic events [Online] / J. Parvizi, A. F. Chen, C. Restrepo [et al.] // Rothman Institute Conference Posters, 2015. - Available from : http://jdc.jefferson.edu/ cgi/viewcontent.cgi?article $=1009 \&$ context $=$ rothinsposters .

18. Higher preoperative patient activation associated with better patient-reported outcomes after total joint arthroplasty / J. Andrawis, S. Akhavan, V. Chan [et al.] // Clin. Orthop. Relat. Res. - 2015. - Vol. 473 (8). - P. 2688-2697. DOI: $10.1007 / \mathrm{s} 11999-015-4247-4$.

19. Singh J. A. The impact of gender, age, and preoperative pain severity on pain after TKA / J. A. Singh, S. Gabriel, D. Lewallen // Clin. Orthop. Relat. Res. — 2008. — Vol. 466 (11). P. 2717-2723. - DOI: 10.1007/s11999-008-0399-9.

20. Prediction and assessment of the severity of post-operative pain and of satisfaction with management / T. Thomas, C. Robinson, D. Champion [et al.] // Pain. — 1998. — Vol. 75 (2-3). P. $177-185$.

21. Della Valle C. J. Thromboembolism after hip and knee arthroplasty: diagnosis and treatment / C. J. Della Valle, D. J. Steiger, P. E. Di Cesare // J. Am. Acad. Orthop. Surg. — 1998. Vol. 6 (6). - P. 327-336.

The article was received at editorial office 10.02.2017

\title{
СУЩЕСТВУЕТ ЛИ ЛУЧШИЙ СПОСОБ ПРОФИЛАКТИКИ ТРОМБОЭМБОЛИИ ВЕН ПОСЛЕ ЭНДОПРОТЕЗИРОВАНИЯ КОЛЕННОГО СУСТАВА?
}

\author{
M. A. Snyder ${ }^{1}$, O. E. Vyrva ${ }^{2}$
}

${ }^{1}$ Ортопедический Центр Высшего Мастерства, Институт Ортопедии и Спортивной медицины «Trihealth», Цинциннати. США

${ }^{2}$ ГУ «Институт патологии позвоночника и суставов им. проф. М. И. Ситенко НАМН Украины», Харьков

$\bowtie$ Oleg Vyrva, MD, Prof.: dr.olegvyrva@gmail.com 